\title{
ARTICLE OPEN Dissecting the hemagglutinin head and stalk-specific IgG antibody response in healthcare workers following pandemic H1N1 vaccination
}

\author{
Sarah M Tete ${ }^{1,2,3}$, Florian Krammer ${ }^{4}$, Sarah Lartey ${ }^{1,2,3}$, Geir Bredholt ${ }^{1}$, John Wood $^{5}$, Steinar Skrede and Rebecca J Cox $^{1,2,3}$
}

Traditionally, neutralising antibodies that are directed to the major surface glycoprotein hemagglutinin (HA) head domain are measured as surrogate correlates of protection against influenza. In addition to neutralization, hemagglutinin-specific antibodies may provide protection by mediating antibody-dependent cellular cytotoxicity (ADCC). During the 2009 pandemic, vaccination induced HA-specific antibodies that were mostly directed to the conserved HA stalk domain. However, the protective role of these antibodies has not been investigated in detail. We quantified the HA head and stalk-specific antibodies, their avidity, ability to neutralise virus and activate natural killer cells in an ADCC assay. We analyzed sera obtained from 14 healthcare workers who had low hemagglutination inhibition (HI) antibody titres at 3 months after pandemic H1N1 vaccination as well as from 22 controls. Vaccination resulted in a HA stalk dominant antibody response in both low responders and controls. Revaccination of low responders, 5 months later, resulted in a boost in antibodies, with HA head-specific antibodies dominating the response. Comparative analysis of head and stalk antibody avidities revealed that stalk-specific antibodies were qualitatively superior. Furthermore, stalk-specific antibodies mediated virus neutralization and had significantly higher ADCC activity than head-specific antibodies. Despite the head and stalk-specific antibodies being lower in low responders, they had comparable antibody avidity, ADCC functionality and neutralising capacity to those of controls who had high $\mathrm{HI}$ titres post-vaccination. Thus, our study has demonstrated that HA stalk-specific antibodies may have an important role in protection through neutralization and ADCC in low responders who do not maintain seroprotective $\mathrm{HI}$ antibodies.

npj Vaccines (2016) 1, 16001; doi:10.1038/npjvaccines.2016.1; published online 28 July 2016

\section{INTRODUCTION}

Influenza pandemics occur at unpredictable intervals when a novel influenza virus arises which can place a major strain on the global healthcare system. These pandemic viruses can cause high levels of severe illness and death. In 2009, an influenza A H1N1 virus strain caused a pandemic that started in Mexico and California then rapidly spread globally. The pandemic H1N1 (H1N1pdm) strain was antigenically distinct from the recently circulating seasonal $\mathrm{H} 1 \mathrm{~N} 1$ strains and the majority of the population was immunologically naïve to this virus.

Annual influenza vaccination is recommended for healthcare workers (HCW) so as to maintain the integrity of the healthcare system, reduce absenteeism and reduce influenza $A$ transmission to vulnerable patients. ${ }^{1}$ Vaccination of HCWs has been shown to protect hospitalised patients as well as decrease influenza-like illness and mortality in residents of care-facilities. ${ }^{2}$ During the 2009 pandemic outbreak, the World Health Organization prioritised $\mathrm{HCW}$ for vaccination. H1N1pdm vaccination studies showed that a single dose of pandemic vaccine elicited protective serum hemagglutination inhibition $(\mathrm{HI})$ titres in adults, including HCW. $^{3-8}$ However, seasonal influenza vaccines did not induce protection against the novel H1N1pdm virus. ${ }^{9,10}$
$\mathrm{HI}$ antibodies are directed to the major surface glycoprotein, hemagglutinin $(\mathrm{HA})$, and are the primary correlate of protection. $\mathrm{HA}$ is synthesised as a precursor, HAO, which is then cleaved by host proteases into disulphide-linked HA1 and HA2 subunits, activating virus infectivity. ${ }^{11}$ Antibodies directed to the HA head domain that is composed of the majority of the HA1 subunit prevent virus attachment to the sialic acids on host cells. These antibodies directed to the immunodominant head of the HA have potent neutralising activity that can be detected by $\mathrm{HI}$ or microneutralization assays. Antibodies directed to the HA stalk domain, primarily composed of $\mathrm{HA} 2$ subunit and the $\mathrm{N}$ - and C-terminal ends of HA1, have other functions, including blocking viral fusion with the host cell and antibody-dependent cellular cytotoxicity (ADCC). ${ }^{12}$

H1N1pdm vaccines preferentially induced HA stalk-specific antibodies. In contrast, seasonal inactivated vaccines induce strain specific antibodies directed to the HA head domain and minimal HA stalk-specific antibodies. ${ }^{13,14}$ Furthermore, HA stalk antibodies are postulated to be boosted most efficiently in individuals previously exposed to HAs whose head domains differ substantially from the infecting novel virus strain. Here, a memory B-cell response is boosted against the conserved $\mathrm{HA}$ stalk domain. ${ }^{15}$ Importantly, HA stalk-specific antibodies are broadly reactive and

\footnotetext{
${ }^{1}$ Department of Clinical Science, The Influenza Centre, University of Bergen, Bergen, Norway; ${ }^{2}$ Department of Clinical Science, KG Jebsen Centre for Influenza Vaccines, University of Bergen, Bergen, Norway; ${ }^{3}$ Department of Research \& Development, Haukeland University Hospital, Bergen, Norway; ${ }^{4}$ Department of Microbiology, Icahn School of Medicine at

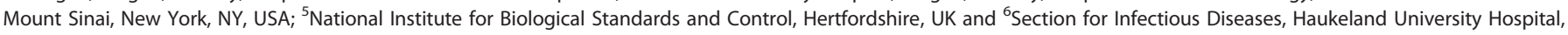
Bergen, Norway.

Correspondence: SM Tete (sarah.tete@k2.uib.no) or RJ Cox (Rebecca.cox@k2.uib.no)
}

Received 7 March 2016; revised 10 May 2016; accepted 18 May 2016 

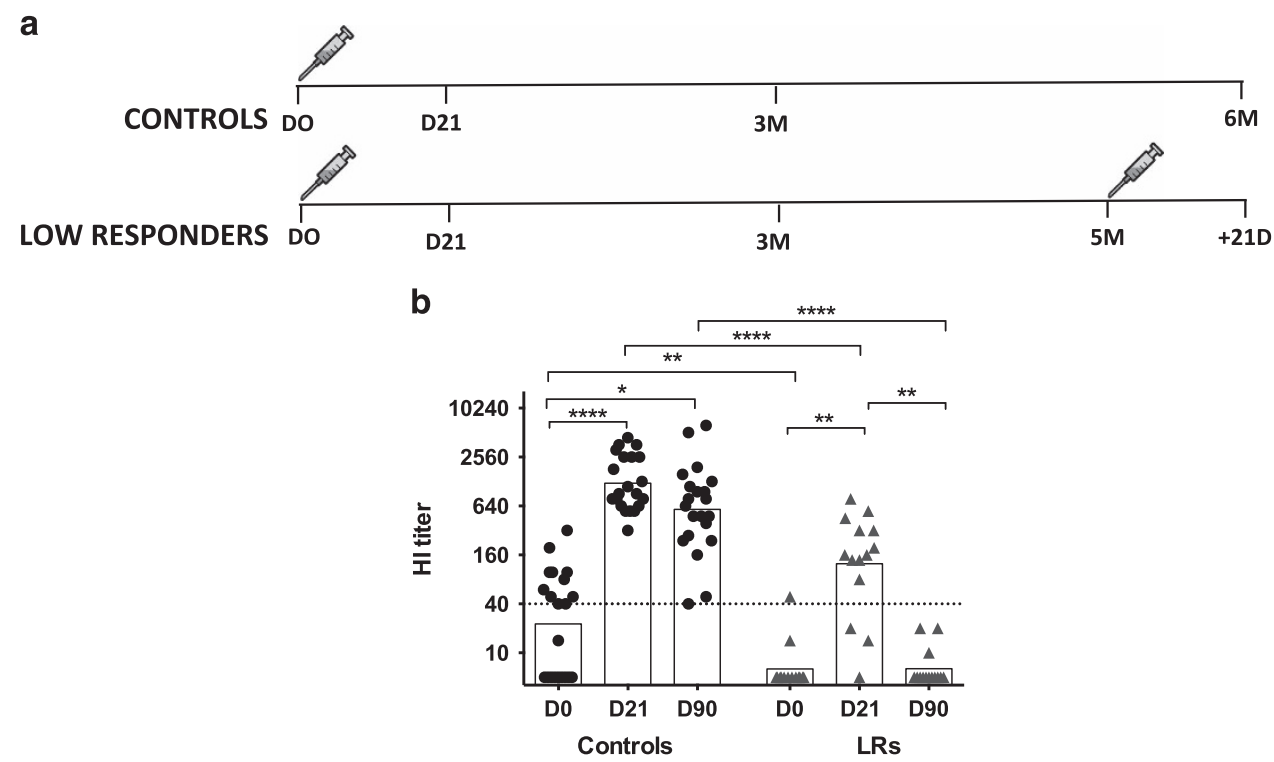

Figure 1. Study outline. (a) Thirty-six HCW received the AS03 adjuvanted monovalent pandemic influenza vaccine in 2009. Serum samples were collected before vaccination (D0); D21, 21 days, 3M and 6M post-vaccination. On the basis of HI titres at 3M, $14 \mathrm{HCW}$ who did not have seroprotective titres (low responders) were offered revaccination. $12 \mathrm{HCW}$ were revaccinated $5 \mathrm{M}$ after the first vaccination. Additional serum samples were collected at time of revaccination (5M) and 21 days later (+D21) in low responders. (b) HI titres against influenza A H1N1pdm virus at D0, D21 and 3M. The dotted line indicates a titre of 40, which is considered protective. * indicates statistically significant differences in responses $P<0.05 ;{ }^{* *} P<0.01 ;{ }^{* *} P<0.001$.

may have a significant role in protection against infection in the absence of HA head-specific antibodies.

In this study, we analyzed the magnitude of HA-specific antibodies induced after adjuvanted pandemic influenza vaccination in HCW. We also analyzed the quality as well as the neutralising and ADCC function of HA-specific antibodies in low-responder HCW who fail to maintain seroprotective $\mathrm{HI}$ responses after $\mathrm{H} 1 \mathrm{~N} 1 \mathrm{pdm}$ vaccination.

\section{RESULTS}

Low responders failed to maintain $\mathrm{HI}$ titres post-vaccination Thirty-six HCW were recruited to the study based on their $\mathrm{HI}$ response and split into two groups; low responders (LRs) who failed to maintain protective $\mathrm{HI}$ titres by 3 months (3M) and a control group (Figure 1a). Fifty per cent of the controls had protective $\mathrm{HI}$ titres (geometric mean titre $(\mathrm{GMT})=23$ ) before vaccination in comparison to $1 \mathrm{LR}(7 \%)(\mathrm{GMT}=6)$. Following pandemic $\mathrm{H} 1 \mathrm{~N} 1$ vaccination, $\mathrm{HI}$ titres increased significantly by D21 in both groups $(P<0.01)$. However, in LRs, titres were significantly lower $(\mathrm{GMT}=132)(P<0.001)$ than controls $(\mathrm{GMT}=$ 1,223). All the controls maintained their protective $\mathrm{HI}$ titres at $3 \mathrm{~mol} / \mathrm{l}$, whereas $\mathrm{HI}$ titres decreased below protective levels for all the LRs (Figure 1b). This decrease may imply that LRs are no longer protected, although their antibodies may mediate protection through other mechanisms.

The back-boost of cross-reactive antibodies to pre-pandemic seasonal H1N1 strains is lower in LRs

The H1N1 strains circulated from 1977 to 2008, when the H1N1pdm appeared and replaced them as the seasonal strain. We examined the cross-reactivity of the $\mathrm{HI}$ response in 20 controls and 14 LRs to the following historical strains; USSR/77, Brazil/78, Taiwan/86, Texas/91, New Caledonia/99 and Brisbane/07 (Figure 2).

Interestingly, pre-vaccination, LRs had significantly lower titres (GMTs $<40)$ to all the seasonal H1N1 strains tested in this study $(P<0.05)$ than the equivalent responses to $\mathrm{A} /$ California/07/09.

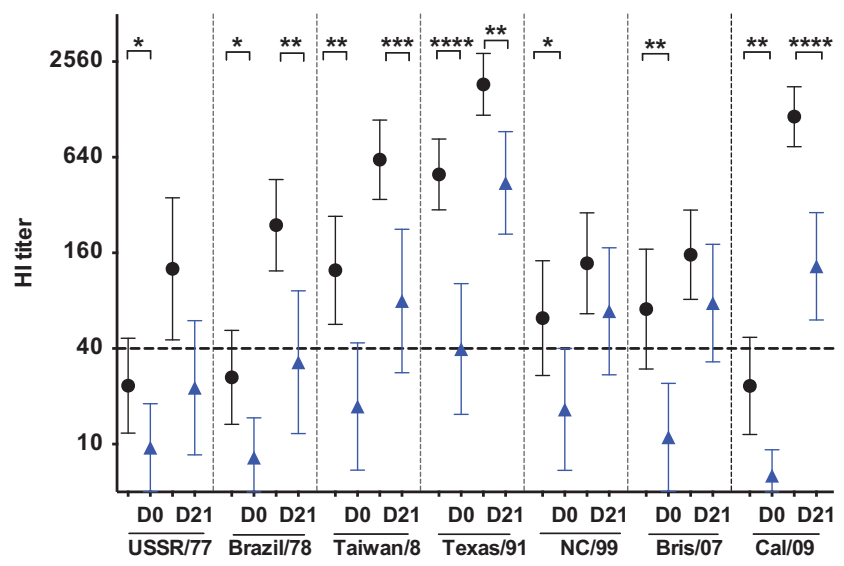

Figure 2. $\mathrm{HI}$ titres to previous seasonal $\mathrm{H} 1 \mathrm{~N} 1$ viruses. The $\mathrm{HI}$ assay was used to examine the cross-reactive $\mathrm{HI}$ responses at D0 and D2 1 against six historical H1N1 virus strains; A/USSR/90/77 (USSR), A/Brazil/11/1978 (Brazil), A/Taiwan/1/86 (Taiwan), A/Texas/36/91 (Texas), A/New Caledonia/20/99 (NC) and A/Brisbane/59/07 (Bris). The $\mathrm{HI}$ response to the $\mathrm{H} 1 \mathrm{~N} 1 \mathrm{pdm}(\mathrm{Cal})$ virus is shown in the last column. The black circles represent the GMTs for controls, whereas the blue triangles represent the GMTs for LRs. The error bars on the plot show the lower and upper extremes. ${ }^{*} P<0.05$; ${ }^{* *} P<0.01$; $* * * P<0.001, * * * * P<0.0001$.

This was in contrast to controls who had $\mathrm{HI}$ GMT $>40$ for all strains except for the oldest strains (USSR/77 and Brazil/78). Of the controls, $70-100 \%$ had seropositive pre-vaccination titres to Taiwan/86, Texas/91, New Caledonia/99 and Brisbane/07. Pre-vaccination sera showed greatest reactivity to Texas/91 in both controls and LRs with GMT of 137 and 40, respectively. Pre-vaccination titres to Texas/91 were higher than those observed to $2009 \mathrm{H} 1 \mathrm{~N} 1 \mathrm{pdm}$.

After pandemic H1N1 vaccination, (D21) sera cross-reacted with all the seasonal $\mathrm{H} 1 \mathrm{~N} 1$ strains in controls with seroprotective titres in $80-100 \%$ of subjects. Back-boosting, with $\mathrm{HI}$ titres greater or comparable to those to H1N1pdm, was induced for Texas/91 and 
a
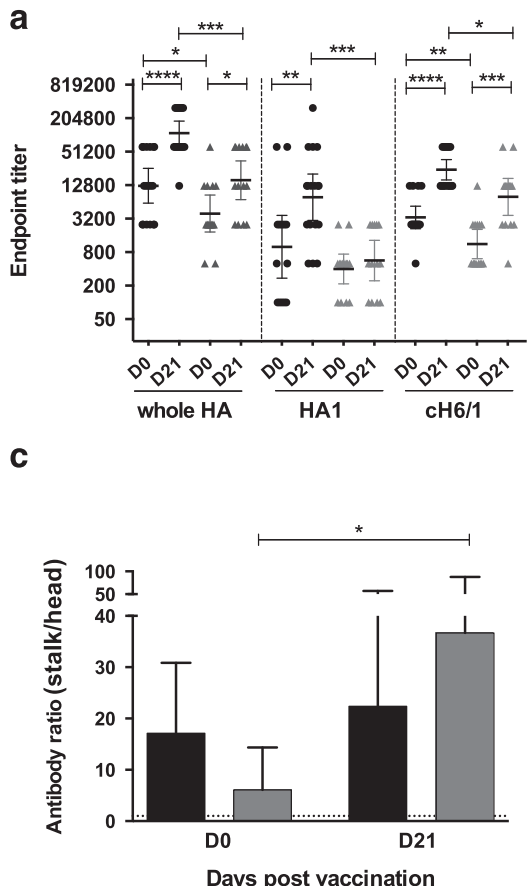

b
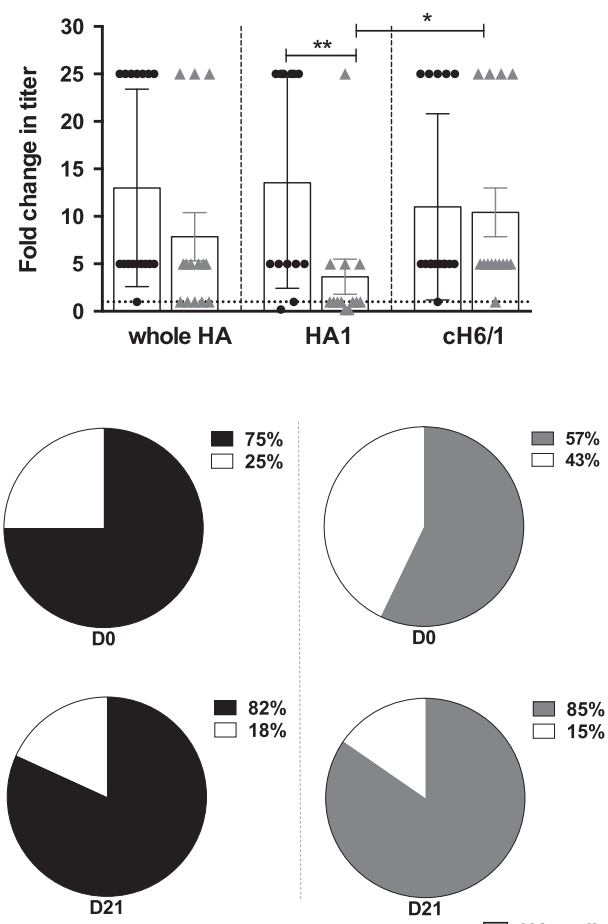

D21 HA stalk dominant

equal head-stalk dominance

Figure 3. Hemagglutinin-specific IgG antibody responses. (a) IgG titres specific to the whole HA (Cal09), HA head domain (HA1) and stalk domain $(\mathrm{CH6/1)}$ at D0 and D21 after vaccination in controls (black circles) and low responders (grey triangles). (b) Fold change in specific lgG titres calculated as D21 titres-D0 titres. (c) Bar chart shows the ratio of HA stalk-specific antibodies to HA head-specific antibodies at D0 and D21. Bars show mean and error bars show s.d. Pie charts with the proportion of controls (black) and LRs (grey) who have stalk dominant lgG antibody responses. The white pieces of the pie show the proportion of HCW with HA head dominated or equal HA head and stalk dominated IgG response. ${ }^{*} P<0.05 ;{ }^{* *} P<0.01 ;{ }^{* * *} P<0.001,{ }^{* * * *} P<0.0001$.

Taiwan/86 with GMTs of 1,841 and 618 , respectively. In comparison to controls, cross-reactive antibodies in LRs were significantly lower to 4 of the 6 strains; USSR/77, Brazil/78, Taiwan/ 86 and Texas/91 $(P<0.05)$. At D21, the GMTs to USSR/77 and Brazil/78 were low at 23 and 33, respectively, whereas for the 4 other seasonal H1N1 strains titres were $>40$ (range: 69-442). Seroprotection was lower in LRs (42-92\%) compared to controls, with the highest seroprotective titres observed for the Texas/91 strain. In summary, these results show that H1N1 cross-reactivity of post-H1N1pdm vaccination sera to seasonal H1N1 strains is reduced in LRs.

HA stalk-specific lgG antibodies dominate the response to pandemic $\mathrm{H} 1 \mathrm{~N} 1$

We dissected the specificity of the antibodies to the different HA domains, which may have different functions in controlling infection. Sera were evaluated for antibodies specific for the H1N1pdm whole HA, HA1 (head domain) and chimeric HA construct, cH6/1 (stalk domain).

Pre-vaccination, antibodies specific to the whole HA were significantly lower in LRs compared to controls $(P<0.05)$. Vaccination resulted in an increase in these antibodies, however to a lesser magnitude than in controls (Figure 3a). The antibody levels induced by vaccination were significantly lower in LRs compared to controls to both the HA head and the stalk domains. In controls, both the HA head and stalk-specific antibodies increased significantly after vaccination $(P<0.01)$. However, in the LRs only stalk-specific antibodies increased significantly $(P<0.001)$, whereas HA head-specific antibodies remained low. The fold increase in HA stalk-specific antibodies was comparable between the two groups, whereas controls had a higher fold increase in HA head-specific antibodies $(P<0.01)$ (Figure $3 b$ ). HA stalk-specific antibodies dominated the response pre- and post-vaccination in both groups (Figure $3 c$ ). There was a significant increase in the proportion of HA stalk antibodies at D21 in comparison to pre-vaccination ratios in LRs $(P<0.05)$. Pre-vaccination, $75 \%$ of controls had HA stalk dominant IgG antibodies, whereas at D21, $82 \%$ had a HA stalk dominant lgG antibodies. However, in LRs, there was an increase from 57 to $85 \%$ in number of participants with stalk dominant antibody responses at D0 and D21, respectively (Figure 3c).

Revaccination of LRs boosts the HA head-specific antibodies Next, we analysed the kinetics of the HA-specific lgG antibodies in LRs following revaccination with the AS03 adjuvanted monovalent pandemic $\mathrm{H} 1 \mathrm{~N} 1$ vaccine. After revaccination, the HA head was no longer novel so we expected a boost of the response to HA head domain. As we did not collect sera from controls at the time of LR revaccination $(5 \mathrm{M})$, we used sera collected at $6 \mathrm{M}$ for comparison purposes. IgG antibodies specific to the whole HA where maintained for at least $6 \mathrm{M}$ in controls but not in LRs where they decreased to pre-vaccination levels. However, revaccination of LRs resulted in a boost in the whole HA-specific antibodies $(P<0.01)$ (Figure 4a).

In controls, the HA head-specific IgG antibodies decreased to pre-vaccination levels by $6 \mathrm{M}$. Similarly, in LRs, HA head-specific IgG antibodies returned to pre-vaccination levels at the time of revaccination (5M). The level of these HA1 specific lgG antibodies was significantly lower in the LR group at $5 \mathrm{M} \quad(P<0.001)$ (Figure $4 b$ ). In contrast to HA head-specific antibodies that decreased by $6 \mathrm{M}$ post-vaccination, HA stalk-specific antibodies were maintained for at least $6 \mathrm{M}$ in controls. However, in LRs, 


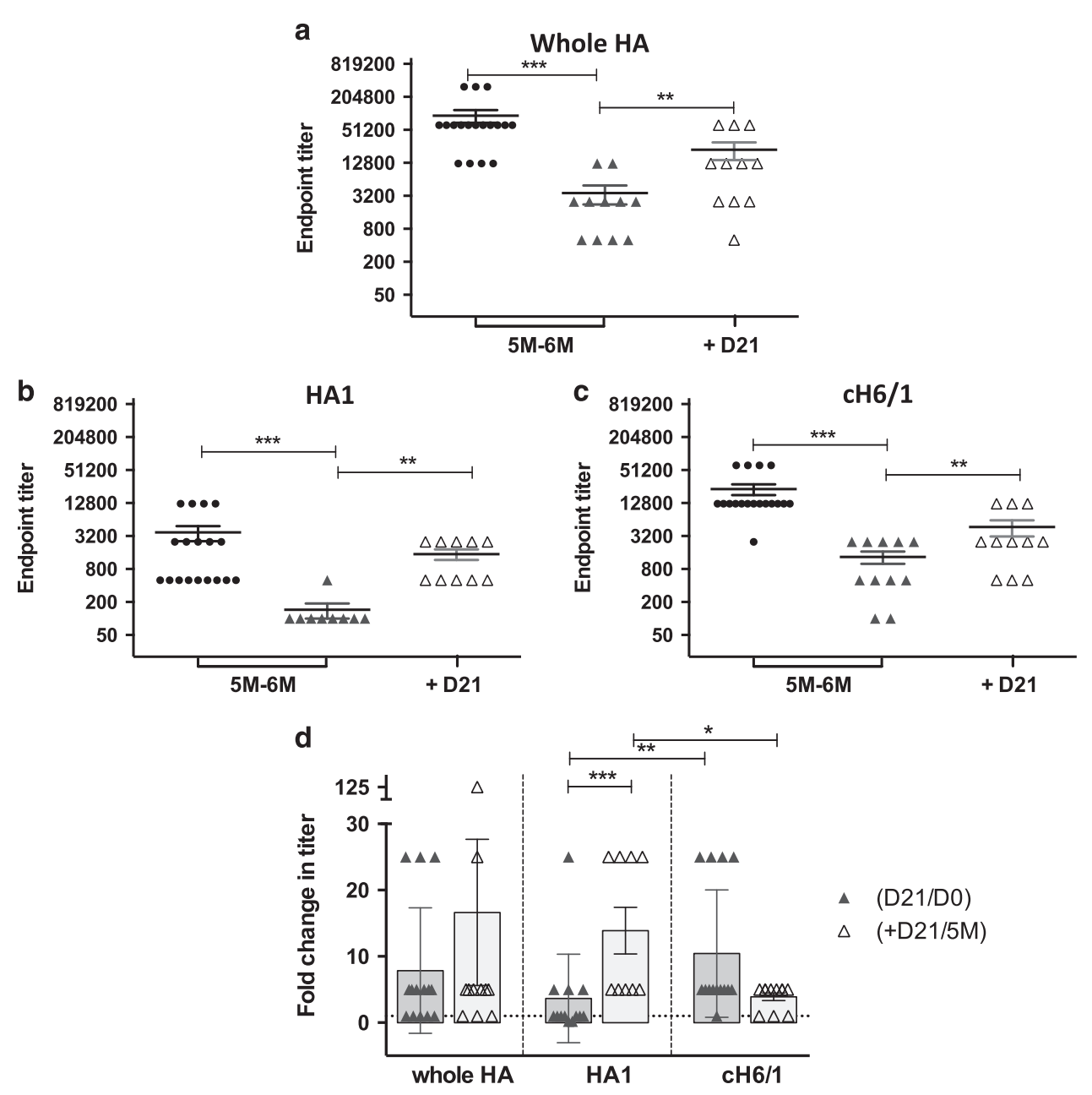

Figure 4. Hemagglutinin-specific lgG antibody responses following revaccination. IgG titres specific to the (a) whole HA, (b) HA head domain and (c) HA stalk domain at 6M in controls (black circles) and 5M (revaccination) (grey triangles) and 21 days after revaccination (+D21) (open triangles) in LRs. (d) The bar chart shows fold induction of IgG titres at D21 post-vaccination over D0 titres. Mean fold induction is shown as bars and grey triangles represent each individual's fold induction after the first vaccination (D21/D0), whereas fold induction 21 days after revaccination is shown in open triangles $(+\mathrm{D} 21 / 5 \mathrm{M})$. ${ }^{* *} P<0.01 ;{ }^{* * *} P<0.001$.

a

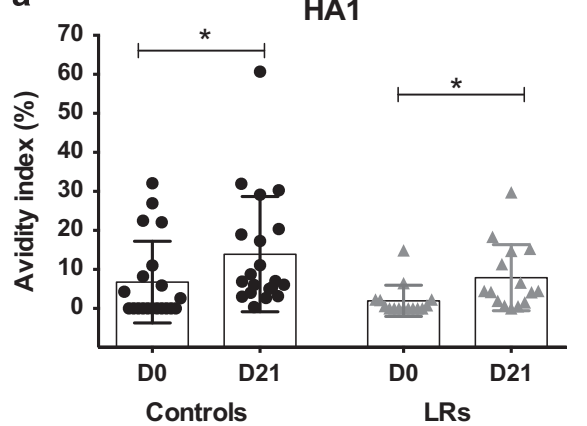

b

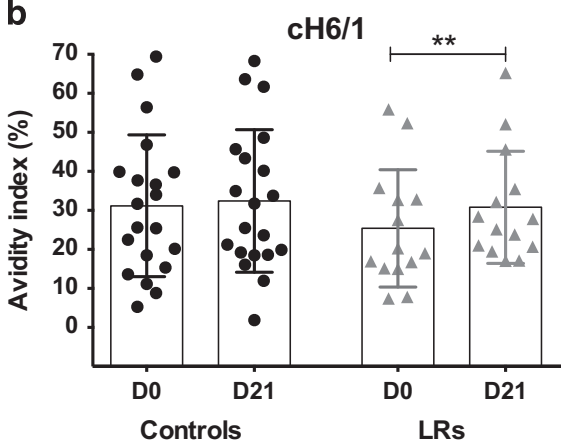

Figure 5. Hemagglutinin-specific IgG antibody avidity. Avidity of (a) HA head domain specific and (b) HA stalk-specific IgG at D0 and D21 post-vaccination. Avidity index was calculated as the percentage of HA-specific lgG antibodies remaining bound after $1.5 \mathrm{nmol} / \mathrm{l}$ NaSCN treatment measured as (absorbance of treated samples $\div$ absorbance of untreated samples) $\times 100 \%$. ${ }^{*} P<0.05 ;{ }^{* * P}<0.01$.

HA stalk-specific antibodies were significantly lower than controls $(P<0.001)$ and decreased to pre-vaccination levels $5 \mathrm{M}$ post-vaccination (Figure $4 c$ ).

Revaccination boosted both the HA head and HA stalk-specific lgG response by D21 $(P<0.01)$. After the first vaccination, the IgG titres increased mostly to the HA stalk, in contrast revaccination induced antibody responses mostly to the HA head (Figure 4d). Twenty-one days after the first vaccination, there was higher fold increase in HA stalk-specific IgG than HA head-specific IgG titres. However, following revaccination, there was a significantly higher fold increase in IgG titres to the HA head than after the first vaccination $(P<0.001)$. 

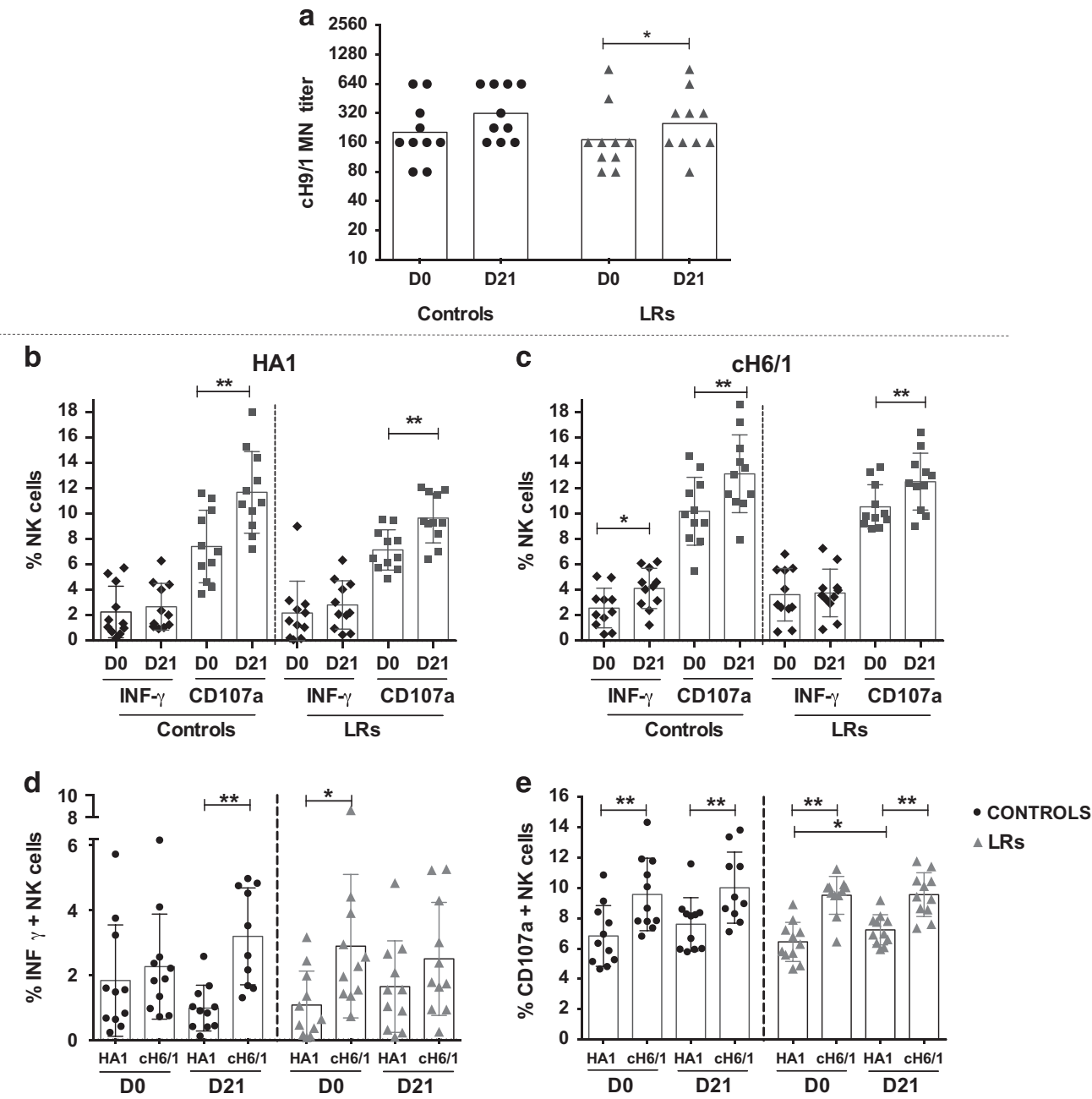

Figure 6. Hemagglutinin-specific antibodies functionality in neutralization and ADCC. (a) H1 HA stalk-specific neutralizing antibodies. Geometric mean titres of neutralizing antibodies against $\mathrm{cH} 9 / 1 \mathrm{~N} 3$ virus with $\mathrm{H} 1 \mathrm{HA}$ stalk domain, irrelevant neuraminidase and $\mathrm{H} 9 \mathrm{HA}$ head domain in 10 controls (circles) and 10 low responders (triangles). The frequency of INF- $\gamma$ expression and CD107a by NK cells to (b) HA head domain (HA1) and (c) stalk domain (CH6/1) in the presence of sera diluted 1:10 at D0 and D21 after vaccination in 10 controls and 10 low responders. (d) INF- $\gamma$ and (e) CD107a expression mediated by HA head-specific antibodies and HA stalk-specific antibodies was compared for each sample sera standardized in an ELISA to give OD of $0.7 \pm 0.2 .{ }^{*} P<0.05 ;{ }^{*} P<0.01$.

HA stalk-specific IgG antibodies show avidity superior to HA head-specific antibodies

To evaluate the quality of the antibodies in the controls and LRs, we measured the avidity of HA head and HA stalk-specific IgG antibodies in these two cohorts of HCW. The avidity of HA-specific lgG antibodies was measured in an avidity enzyme-linked immunosorbent assay (ELISA) using NaSCN as a chaotropic agent. Untreated sera were compared to those treated with $1.5 \mathrm{~mol} / \mathrm{l}$ $\mathrm{NaSCN}$, and the percentage of bound IgG antibodies remaining after $1.5 \mathrm{~mol} / \mathrm{l} \mathrm{NaSCN}$ treatment was calculated. At baseline, the avidity of head-specific antibodies was low. The avidity of head-specific antibodies increased significantly in both controls and LRs following vaccination $(P<0.05)$ (Figure $5 a)$. In controls, the avidity index increased from a mean of $3.02-7.11 \%$ at D21. In LRs, an increase from a mean of $1.95-8.14 \%$ was observed post-vaccination.

The avidity of HA stalk-specific antibodies was significantly higher than that of HA head-specific antibodies at both timepoints (Figure $5 \mathrm{~b}$ ). In controls, the \% of IgG antibodies remaining bound after NaSCN treatment was $25.74 \%$ (range: $5.3-69 \%$ ) at baseline. Vaccination resulted in a significant increase in the avidity of HA stalk-specific IgG antibodies in LRs only. The avidity index of the IgG antibodies increased from $25.40 \%$ (range:
7.46-55.92\%) at baseline to $30.82 \%$ (range: $17.13-65.26 \%$ ) at D21 post-vaccination in LRs $(P<0.01)$. Despite, the quantity of the stalk-specific antibodies being lower in LRs compared to controls, the antibodies displayed equivalent avidity at both time-points.

HA stalk-specific antibodies can neutralise virus in the absence of $\mathrm{HI}$ antibodies

To assess the in vitro functionality of $\mathrm{H} 1 \mathrm{HA}$ stalk-specific antibodies, we performed a microneutralization assay with a virus that expresses a $\mathrm{cH} 9 / 1 \mathrm{HA}$ and an irrelevant $\mathrm{N} 3$ neuraminidase. The HA stalk domain of this virus is derived from $\mathrm{H} 1$, and the $\mathrm{HA}$ head domain is from $\mathrm{H} 9$. Subjects in this study are expected to be naive to the avian $\mathrm{H} 9$ and $\mathrm{N} 3$ in this virus. No standard virus neutralization titre for $50 \%$ protection has been recognised. However, a previous study in H1N1pdm-infected adults showed that the MN titre was generally twofold higher than the $\mathrm{HI}$ titre when the $\mathrm{HI}$ titre was $\leqslant 160 .{ }^{16}$ We therefore used a threshold of 80 to define protective titres. H1 HA stalk-specific neutralising antibodies were detected pre-vaccination when the majority of controls (50\%) and LRs (93\%) had HI titres < 40 (Figure 6a). These $\mathrm{H} 1 \mathrm{HA}$ stalk-specific neutralising antibodies were present in all subjects with GMT of 204 and 171.5 in controls and LRs, 
respectively. Vaccination resulted in a significant increase in HA stalk-specific neutralising antibodies in LR $(P<0.05)$ but not in controls.

HA stalk-specific antibodies are better mediators of natural killer (NK) cell activation

As the HA-specific antibody response was dominated by HA stalk-specific antibodies, we wanted to determine the functionality and possible protective mechanisms of these antibodies, especially in LRs where HA head-specific antibodies were low. We therefore assessed the ADCC induced by HA head and HA stalk-specific antibodies using an NK activation assay measuring CD107a (degranulation) and INF- $\gamma$ expression by flow cytometry. The gating strategy for flow cytometry is shown in Supplementary Figure S1. We used sera diluted 1:10 rather than endpoint titres as a positive correlation between NK cell activation at a single sera dilution of 1:10 and NK activation end point titre has been previously shown. ${ }^{17}$ Pre-vaccination, NK cell activation was mediated by both HA head and HA stalk-specific antibodies and the levels of NK cell activation were comparable between LRs and controls. Vaccination resulted in increased NK cell activation with a significant increase in head and stalk antibody-mediated CD107a expression being observed for both LRs and controls $(P<0.01)$ (Figure $6 \mathrm{~b}, \mathrm{c}$ ). However, the levels of INF- $\gamma$ expression mediated by HA head-specific antibodies did not increase for either LRs or controls. In contrast to controls who had an increase in INF- $\gamma$ expression at D21, no change in HA stalk antibody-mediated INF- $\gamma$ expression was detected in LRs (Figure 6c).

In order to make a direct comparison of the levels of head versus stalk antibody-mediated NK cell activation, we standardized sera in an ELISA assay to give an optical density (OD) of $0.7 \pm 0.2$. In controls, pre-vaccination $\mathrm{HA}$ head and HA stalk-specific antibodies induced similar NK cell expression of INF- $\gamma$ (Figure 6d). However, post-vaccination, HA stalk-specific antibodies induced significantly higher INF- $\gamma$ expression than head-specific antibodies in controls $(P<0.01)$. In contrast, LRs' HA stalk-specific antibodies induced significantly higher INF- $\gamma$ expression at D0 than head-specific antibodies $(P<0.05)$, whereas at D21 the INF- $\gamma$ expression levels were maintained. Prevaccination, HA stalk-specific antibodies induced significantly higher NK cell CD107a expression than HA head-specific antibodies in both LRs and controls (Figure 6e). Post-vaccination, only HA head-specific antibodies from LRs had an increased ADCC activity as measured by CD107a expression. Despite no increase in CD107a expression at D21, the HA stalk-specific antibodies maintained a significantly higher ADCC induction than HA head-specific antibodies in both LRs and controls $(P<0.01)$. Furthermore, there was no significant difference in the ADCC mediated by both head and stalk-specific antibodies between LRs and controls. Interestingly, there was no correlation between NK cell activation and $\mathrm{HI}$ titres in controls (data not shown). However, in LRs, $\mathrm{HI}$ titres negatively correlated with NK cell INF- $\gamma$ expression mediated by both HA head and HA stalk antibodies $(P<0.01)$. This suggests that H1N1pdm vaccination induced HA-specific antibodies that can mediate FcyR-dependent NK cell activation, regardless of $\mathrm{HI}$ antibodies.

\section{DISCUSSION}

The H1N1pdm virus contained a novel HA head domain that was different from the pre-pandemic seasonal $\mathrm{H} 1$ viruses. Pandemic vaccination induced antibodies that were directed towards the immunosubdominant conserved epitopes on the HA stalk domain. $^{13,14}$

In the current study, we aimed to investigate and to increase understanding of the HA-specific IgG responses of LR HCW following 2009 pandemic influenza A (H1N1) vaccination. We used chimeric HA constructs to differentiate between the response to the HA head and stalk domains as these antibodies may have different functions in controlling influenza infection. Influenza specific responses are commonly measured by the $\mathrm{HI}$ assay. $\mathrm{HI}$ antibodies are a correlate of protection, and are mostly directed to the HA head domain and do not necessarily reflect the entire spectrum of vaccine induced antibodies. We demonstrated that LRs IgG antibodies were quantitatively inferior to controls but qualitatively similar.

We showed that a single dose of $\mathrm{ASO} 3$ adjuvanted pandemic $\mathrm{H} 1 \mathrm{~N} 1$ vaccine elicited a significant $\mathrm{HI}$ response in controls that was maintained by $3 \mathrm{M}$ post-vaccination, whereas in LRs the response was lower and waned by $3 \mathrm{M}$. The lower $\mathrm{HI}$ response may be attributed to the lower quantities of HA head-specific antibodies in LRs as antibodies measured by the $\mathrm{HI}$ assay are predominantly HA head-specific.

Back-boosting, where a substantial response to older viruses is induced, depending on pre-exposure has been recently described for both H3N2 and H1N1 viruses. ${ }^{18,19}$ Li et al. ${ }^{18}$ showed sera from H1N1pdm-infected people had considerable cross-reactivity with H1N1 strains from 1984 to 1994 . They showed that the back-boost for H1N1 was due to a shared epitope in the head domain at H1N1pdm and most seasonal H1N1 strains from 1983 to 1996. HAs of most seasonal H1N1 between 1983 and 1996 contained a K133 amino acid at Sa antigenic site of HA, but not H1N1 viruses before 1983 or after 1996. Our results are in agreement with this as we showed back-boost of $\mathrm{HI}$ against viruses up to Texas/91 but not much against NC/99 and Brisbane/07. LRs had a lower backboost of cross-reactive $\mathrm{HI}$ antibodies to pre-pandemic, seasonal H1N1 strains. This may be due to differences in exposure history to different $\mathrm{H} 1 \mathrm{~N} 1$ strains or due to limited induction of $\mathrm{HI}$ antibodies that bind to shared epitopes on the HA of pandemic and seasonal H1N1 strains. Moreover, the pandemic vaccine may have elicited antibodies with broader specificities that bind the same epitopes as antibodies induced by seasonal $\mathrm{H} 1 \mathrm{~N} 1$ strains as well as to additional epitopes. Despite the lower $\mathrm{HI}$ antibodies detected in the LR cohort, HA stalk-specific antibodies may also provide an alternative method of protection.

We tested for the HA domain binding of IgG antibodies and found that HA stalk-specific antibodies were lower pre-vaccination in LRs, possibly due to differences in the priming or infection history between controls and LRs or due to the characteristics of the HA-specific memory previously generated. Even though pre-vaccination HA stalk-specific antibodies were lower, a single dose of H1N1pdm vaccine elicited significant stalk-specific antibodies more efficiently than HA head-specific antibodies. However, 5M post-vaccination, HA head and stalk-specific antibodies in LRs had decreased and were significantly lower than those in controls. Although none of the HCW reported H1N1pdm infection, it cannot be ruled out that some controls had exposure or subclinical infection between the $5 \mathrm{M}$ and $6 \mathrm{M}$ interval. Alternatively, the lower antibodies in LR could be explained by poor antibody maintenance in this cohort. The decreased antibody titres may not necessarily mean that the HCW were no longer protected, as factors other than antibody titres may be important in long-term protection.

These findings raise questions as to: (i) why the conserved HA stalk domain shows superior immunogenicity after H1N1pdm vaccination, whereas after seasonal vaccination the HA headspecific response dominates ${ }^{20,21}$; and (ii) the effect of homologous boosting on HA domain binding. We found a significantly higher boost in head than stalk-specific antibodies following revaccination of LRs. This is in line with previous reports following H1N1pdm and H5N1 vaccination. ${ }^{22,23}$ Ellebedy et al. demonstrated that the first dose of $\mathrm{H} 5 \mathrm{~N} 1$ vaccination elicited a stronger stalk-specific response than head-specific response. However, booster vaccination resulted in a vigorous head-specific response and marginal increase in stalk-specific antibodies. ${ }^{23}$ These findings 
support the theory that at the time of the first vaccination, vaccinees had negligible pre-existing memory $B$ cells specific to the immunodominant HA head domain in comparison with stalkspecific ones. The strong stalk-specific antibody response likely reflects the reactivation of stalk-specific memory $B$ cells generated by previous seasonal H1N1 infections. Accordingly, memory B cells specific for the immunosubdominant $\mathrm{HA}$ stalk were recruited and reactivated in the absence of competition from memory $B$ cells specific for the immunodominant HA head domain. A primary antibody response was also induced to the HA head domain resulting in the increase in plasmablasts and memory $B$ cells generation. Upon booster vaccination, the recently generated head-specific memory B cells out-competed the stalk-specific memory B cells. ${ }^{24}$

The quality of antibodies is important for their functionality. In our analysis, HA stalk-specific antibodies not only dominated the response but also displayed superior avidity to head-specific antibodies. These results are in agreement with the findings of He et al., ${ }^{20}$ who showed that plasmablast-derived polyclonal antibodies from elderly ( $>70$ years) after $\mathrm{H} 1 \mathrm{~N} 1 \mathrm{pdm}$ vaccination had higher avidity than those from young (18-32 years) and that HA2 specific antibodies had higher avidity than HA1 specific antibodies. The higher avidity of stalk-specific antibodies could be explained by antibody secreting cells producing these antibodies being derivatives of memory $B$ cells from previous H1N1 encounters that have gone through several rounds of selection and affinity maturation. ${ }^{25,26} \mathrm{As}$ the 2009 pandemic H1N1 contained a novel head, different from the pre-pandemic seasonal H1N1 viruses, most of the HA head-specific antibodies would have been generated from antibody secreting cells from a primary immune response that contain few somatic mutations, explaining the lower avidity of HA head-specific antibodies. It has been shown that broadly cross-reactive HA-specific antibodies that exhibit high levels of somatic hypermutation are induced after H1N1pdm vaccination or infection. ${ }^{13,27}$ Analysis of the somatic mutation status of the immunoglobulin genes from the memory $B$ cells or plasmablasts specific for the HA head and stalk domains is required to give insight into the origin of the response in our cohort of HCW. These high-avidity HA stalk-specific antibodies may have an important role in protecting these LRs in the absence of $\mathrm{HI}$ antibodies. However, a correlate of protection for these antibodies remains to be determined.

We showed that high titres neutralising HA stalk-specific antibodies were present in all subjects pre-vaccination, when $\mathrm{HI}$ antibodies were absent or low in the majority of the HCW. The neutralising stalk-specific antibodies were maintained in controls and only increased significantly in LRs after vaccination. These neutralising stalk-specific antibodies could be protective regardless of the $\mathrm{HI}$ titre and could have a significant role in protecting the low responders who have significant quantities of HA stalk-specific antibodies.

In addition to virus neutralization, HA-specific antibodies can bind to infected cells and activate NK cells through FcyR resulting in lysis of target cells and secretion of cytokines like INF-.$^{17}$ In this study, we showed that ADCC antibodies were present pre-vaccination when the majority of the subjects were $\mathrm{HI}$ seronegative and these ADCC antibodies were mostly directed towards the HA stalk domain. These pre-existing ADCC antibodies may assist in clearance of $\mathrm{H} 1 \mathrm{~N} 1 \mathrm{pdm}$ virus infection. Post-vaccination, the HA stalk-specific antibodies were better mediators of ADCC than HA head-specific antibodies. This difference in the ADCC activity between HA head and stalk-specific antibodies may be attributed to the relative difference in affinities of these antibodies for HA. Despite LRs having lower post-vaccination $\mathrm{HI}$ titres and lower HA-specific lgG titres than controls; their antibodies had comparable ability to mediate NK cell activation. We found no correlation between $\mathrm{HI}$ titre and NK cell activation in controls, whereas in LRs INF- $\gamma$ expression negatively correlated with $\mathrm{HI}$ titres. Our results are similar to a previous report that found no correlation between $\mathrm{HI}$ titres and HA-specific ADCC antibodies. ${ }^{17}$ However, these authors characterised the response induced by antibodies that bound to the whole HA protein, whereas we dissected between HA head and stalk-specific antibodies. Of note, the $\mathrm{HI}$ and ADCC assays measure different aspects of antibody function. HI assay assesses the binding of the antibody through its Fab region to antigen, whereas the ADCC assay assesses the NK activation by antibodies bound through their Fc region. Fc-FcyR interactions are required for the protection by HA stalk-specific antibodies suggesting a role of ADCC antibodies in protection. ${ }^{28-30}$ Furthermore, cross-reactive ADCC antibodies to influenza virus have been reported in the absence of neutralising antibodies. ${ }^{31}$ Thus, it is likely that HA stalk-specific antibodies may provide alternative protection in this cohort of LRs where HA head-specific antibodies are low and induced short-term protection as measured by the $\mathrm{HI}$ assay. This underlines a possible limitation of using $\mathrm{HI}$ titre as a sole predictive correlate of protection.

In summary, the characterisation of the HA stalk-specific antibodies denotes an important step in understanding the protective capacity of these antibodies. Further analysis of the HA stalk-specific antibody repertoire and function would facilitate rational vaccine design. In LRs where the $\mathrm{HI}$ responses are poor, vaccination strategies could potentially aim to induce $\mathrm{HA}$ stalk-specific antibodies. The lower quantity of HA-specific antibodies could be compensated for by the higher avidity of HA stalk-specific antibodies that have better ADCC functionality as well as neutralising capacity. Enhancing the amount of HA stalk-specific antibodies elicited by vaccination or booster vaccination should be considered in LR HCW. These antibodies could provide broad cross-protection and could be used for immunological priming of the general population to quickly respond to a future pandemic influenza threat.

\section{MATERIALS AND METHODS}

Study design and participants

The HCWs were vaccinated in October 2009 at the Haukeland University Hospital ( $\mathrm{HUH}$, Bergen, Norway) with a single dose of the monovalent pandemic H1N1 vaccine (Pandemrix) adjuvanted ASO3 (GlaxoSmithKline (GSK), Wavre, Belgium).

Thirty-six HCW were retrospectively selected by their $\mathrm{HI}$ antibody response at $3 \mathrm{M}$ after pandemic $\mathrm{H} 1 \mathrm{~N} 1$ vaccination. On this basis, a cohort of 14 LRs who failed to respond or maintain a protective $H$ l antibody response $($ titre $\geqslant 40)$ at $3 \mathrm{M}$ after vaccination were selected. As a control group, $22 \mathrm{HCW}$ who maintained a protective $\mathrm{HI}$ antibody response were selected. LRs were offered a second dose of vaccine, and 12 participants were revaccinated $5 \mathrm{M}$ later. Sera were collected at vaccination, day 21 (D21), 3M, and $6 \mathrm{M}$ post-vaccination in controls. In revaccinated LRs, additional sera were collected at revaccination $(5 \mathrm{M})$ and 21 days later (Figure 1a). The inclusion and exclusion criteria for this study are described elsewhere. ${ }^{7}$ All participants provided written informed consent before inclusion in the study, which had ethical and regulatory approval (ClinicalTrials.gov NOT01003288).

\section{HI assay}

Serum samples were treated with receptor destroying enzyme and run in the $\mathrm{HI}$ assay twice in duplicate using turkey red blood cells as previously described. ${ }^{7} \mathrm{HI}$ responses were analyzed against the homologous pandemic $\mathrm{H} 1 \mathrm{~N} 1$ virus strain, $\mathrm{A} / \mathrm{California} / 07 / 09$, and against six prototype seasonal H1N1 strains; A/USSR/90/77, A/Brazil/11/78, A/Taiwan/1/86, A/Texas/36/91, $A /$ New Caledonia/20/99 and $A / B$ risbane/59/07. Seroprotection was defined as an $\mathrm{HI}$ titre $\geqslant 40$. Titres $<10$ were assigned a value of 5 for calculation purposes.

\section{Anti-HA IgG ELISA}

Sera were evaluated in duplicate for IgG antibodies. ${ }^{32}$ The plates were coated with influenza whole HA, HA1 (A/California/06/2009(H1N1)) 
hexahistidine-tagged (eEnzyme, IA-01SW-005P) or cH6/1, a chimeric HA ( $\mathrm{cHA}$ ) that combines $\mathrm{H} 1$ stalk domain with globular head domain derived from $\mathrm{H} 6$ influenza A virus. ${ }^{33}$ The antibody concentrations were calculated as endpoint titres that were determined when the reactivity of the diluted sample reached background levels.

\section{Anti-HA lgG avidity ELISA}

Sera were evaluated for avidity of antibodies against influenza HA1 (A/California/06/2009(H1N1)) hexahistidine-tagged (eEnzyme, Gaithersburg, MD, USA) and $\mathrm{CH} 6 / 1 .^{34}$ Sera were first diluted in duplicate to the appropriate $\mathrm{OD}$ of $0.7 \pm 0.3$ in a direct ELISA and $1.5 \mathrm{~mol} / \mathrm{l}$ sodium thiocyanate (NaSCN) (Sigma, St Louis, MO, USA) was added $1 \mathrm{~h}$ after the sera, followed by $1 \mathrm{~h}$ incubation. The avidity index calculated as: $\left(\mathrm{OD}_{450}\right.$ treated serum $/ \mathrm{OD}_{450}$ untreated serum) $\times 100 \%$.

\section{Virus neutralization assay}

Expansion of MDCK cells. Madin-Darby Canine Kidney (MDCK) (ATCC, Manassas, VA, USA, CCL-34) were cultivated in DMEM supplemented with $10 \%$ fetal bovine serum (FBS) and $1 \times$ Penicillin/Streptomycin. MDCK cells in log-phase growth were plated in 96-well plates such that they are 70$90 \%$ confluent at the time of inoculation. Serum samples were heatinactivated at $56^{\circ} \mathrm{C}$ for $30 \mathrm{~min}$ and run in duplicate. The serum samples were then diluted 2 fold in virus growth medium containing Dulbecco's Modified Eagle's Medium with tosyl phenylalanyl chloromethyl ketonetrypsin, $0.14 \%$ bovine serum albumin, 100 units $/ \mathrm{ml}$ penicillin, $100 \mu \mathrm{g} / \mathrm{ml}$ streptomycin and $0.25 \mu \mathrm{g} / \mathrm{ml}$ amphotericin B. Chimeric 9/1N3 virus was diluted to $50 \%$ tissue culture infectious dose (TCID50) of 100 per $50 \mu \mathrm{l}$ in virus growth medium. Fifty microliters of diluted sera was incubated with $50 \mu \mathrm{l}$ of virus for $1 \mathrm{~h}$ at $37^{\circ} \mathrm{C}$. MDCK cells were washed once with phosphate-buffered solution (PBS) and $100 \mu \mathrm{l}$ of serum-virus mixture was added to the cells. Cells were incubated at $37^{\circ} \mathrm{C}$ for $1 \mathrm{~h}$ then washed once with PBS before $50 \mu \mathrm{l}$ of diluted serum and $50 \mu \mathrm{l}$ of virus growth medium were added to each well. Infected MDCK cells were incubated for $72 \mathrm{~h}$ at $37^{\circ} \mathrm{C}$. Fifty microliters of the supernatant was transferred to a 96-well $\mathrm{V}$ bottom plate and $50 \mu \mathrm{l}$ of $0.7 \%$ turkey red blood cells added. Hemagglutination activity was measured to detect the endpoint of agglutination.

\section{ADCC NK cell activation assay}

The ADCC assay measuring intracellular NK cell IFNY and CD107a expression was conducted as previously described with minor modifications. ${ }^{35}$ Briefly, 96-well plates were coated overnight at $4{ }^{\circ} \mathrm{C}$ with $1 \mu \mathrm{g} / \mathrm{ml} \mathrm{HA} 1$ (A/California/06/2009(H1N1)) $6 \times$ His tagged (eEnzyme, USA) or chimeric $\mathrm{CH} 6 / 1$ in PBS. Plates were washed with PBS and incubated with heat-inactivated human sera for $2 \mathrm{~h}$ at $37^{\circ} \mathrm{C}$. After washing, $10^{5} \mathrm{CD} 16176 \mathrm{v}$ NK-92 cells (mycoplasma-free, human NK cell line expressing high affinity 176 V variant CD16 receptor) (kindly provided by Fox Chase Cancer Center, Philadelphia, PA, USA) were added per test well. As a negative control for each sample, NK-92 cells (lacking expression of CD16) were added to an additional well. The cells were incubated for $16 \mathrm{~h}$ at $37^{\circ} \mathrm{C}$ with antiCD107a-AF488 antibody (Biolegend, San Diego, CA, USA, 328610), Brefeldin A $(5 \mu \mathrm{g} / \mathrm{ml}, \mathrm{BD})$ and monensin $(5 \mu \mathrm{g} / \mathrm{ml}, \mathrm{BD})$. Cells were stained with LIVE/DEAD Fixable Aqua dead cell staining kit (Invitrogen, Carlsbad, CA, USA), anti-CD3-PE CF594 (BD, Franklin Lakes, NJ, USA, 562280) and antiCD56-APC (BD, 555518) before intracellular staining with anti-IFN- $\gamma-B V-421$ (Biolegend, 502532). Cells were acquired on BD Fortessa (San Jose, CA, USA). Data analysis was done using FlowJo version 10 (treeStar, Ashland, OR, USA).

\section{Statistics}

Two-tailed unpaired nonparametric Mann-Whitney tests and paired nonparametric Wilcoxon tests were performed using GraphPad Prism 6 (Graphpad, La Jolla, CA, USA). A P-value $<0.05$ was considered statistically significant.

\section{ACKNOWLEDGEMENTS}

We thank the Healthcare workers at Haukeland University Hospital for participating in this study. We also thank Dr Per Espen Akslesen and study nurses at the Bergen Clinical Vaccine Consortium, Haukeland University Hospital; Marianne Sævik, Hanne Søyland and Astrid Rykkje Heien. The study was funded by the Influenza Centre at the University of Bergen and through funding from the Norwegian Directorate of
Health. The Influenza Centre is funded by the Ministry of Health and Care Services, Norway, the Norwegian Research Council Globvac programme (220670/H10), the European Union (Univax 601738), Helse Vest and the K.G. Jebsen Centre for Influenza Vaccines. The funders had no role in study design, data collection and interpretation, or the decision to submit the work for publication.

\section{CONTRIBUTIONS}

S.M.T. performed the experiments, was involved in interpreting the data and prepared the manuscript. F.K. provided the recombinant HA used in the experiments and was involved in analysis and interpreting the data. S.L. performed some of the experiments and contributed to interpretation of the results. G.B contributed to experimental design and interpretation of results. J.W., S.S. and R.J.C. contributed to the study design, protocol design and interpretation of the results. All authors critically reviewed the manuscript and approved the final article. S.M.T. and R.J.C. are the guarantors.

\section{COMPETING INTERESTS}

F.K. received support from NIAID through U19 Al109946 and the Centers of Influenza Virus Research and Surveillance (CEIRS) contract HHSN272201400008C. The remaining authors declare no conflict of interest.

\section{REFERENCES}

1. Poland, G. A., Tosh, P. \& Jacobson, R. M. Requiring influenza vaccination for health care workers: seven truths we must accept. Vaccine 23, 2251-2255 (2005).

2. Lemaitre, M. et al. Effect of influenza vaccination of nursing home staff on mortality of residents: a cluster-randomized trial. J. Am. Geriatr. Soc. 57, 1580-1586 (2009).

3. Roman, F. et al. Effect on cellular and humoral immune responses of the AS03 adjuvant system in an A/H1N1/2009 influenza virus vaccine administered to adults during two randomized controlled trials. Clin. Vaccine Immunol. 18, 835 (2011).

4. Nicholson, K. G. et al. Safety and antigenicity of non-adjuvanted and MF59-adjuvanted influenza A/Duck/Singapore/97 (H5N3) vaccine: a randomised trial of two potential vaccines against H5N1 influenza. Lancet 357, 1937-1943 (2001).

5. Clark, T. W. et al. Trial of 2009 influenza A (H1N1) monovalent MF59-adjuvanted vaccine. N. Engl. J. Med. 361, 2424 (2009).

6. Zhou, Y. et al. Seroprevalence of pandemic H1N1 antibody among health care workers in Hong Kong following receipt of monovalent 2009 H1N1 influenza vaccine. PLOS ONE 6, e27169 (2011).

7. Madhun, A. S. et al. An adjuvanted pandemic influenza H1N1 vaccine provides early and long term protection in health care workers. Vaccine 29, 266-273 (2010).

8. Greenberg, M. E. et al. Response to a monovalent 2009 influenza A (H1N1) vaccine. N. Engl. J. Med. 361, 2405-2413 (2009).

9. Centers for Disease Control and Prevention. Serum cross-reactive antibody response to a novel influenza $A(\mathrm{H} 1 \mathrm{~N} 1)$ virus after vaccination with seasonal influenza vaccine. MMWR Morb. Mortal. Wkly. Rep. 58, 521-524 (2009).

10. Hancock, K. et al. Cross-reactive antibody responses to the 2009 pandemic H1N1 influenza virus. N. Engl. J. Med. 361, 1945-1952 (2009).

11. Lazarowitz, S. G. \& Choppin, P. W. Enhancement of the infectivity of influenza A and $B$ viruses by proteolytic cleavage of the hemagglutinin polypeptide. Virology 68, 440-454 (1975).

12. Krammer, F. \& Palese, P. Universal influenza virus vaccines: need for clinical trials. Nat. Immunol. 15, 3-5 (2014).

13. Wrammert, J. et al. Broadly cross-reactive antibodies dominate the human B cell response against 2009 pandemic H1N1 influenza virus infection. J. Exp. Med. 208181 (2011).

14. Pica, N. et al. Hemagglutinin stalk antibodies elicited by the 2009 pandemic influenza virus as a mechanism for the extinction of seasonal H1N1 viruses. Proc. Natl Acad. Sci. USA 109, 2573-2578 (2012).

15. Palese, P. \& Wang, T. T. Why do influenza virus subtypes die out? A hypothesis. Mbio 2 (2011).

16. Veguilla, V. et al. Sensitivity and specificity of serologic assays for detection of human infection with 2009 pandemic H1N1 virus in U.S. populations. J. Clin. Microbiol. 49, 2210-2215 (2011).

17. Jegaskanda, S. et al. Age-associated cross-reactive antibody-dependent cellular cytotoxicity toward 2009 pandemic influenza A virus subtype H1N1. J. Infect. Dis. 208, 1051-1061 (2013). 
18. Li, Y. et al. Immune history shapes specificity of pandemic H1N1 influenza antibody responses. J. Exp. Med. 210, 1493-1500 (2013).

19. Fonville, J. M. et al. Antibody landscapes after influenza virus infection or vaccination. Science 346, 996-1000 (2014).

20. He, X. S. et al. Heterovariant cross-reactive B-cell responses induced by the 2009 pandemic influenza virus A subtype H1N1 vaccine. J. Infect. Dis. 207, 288-296 (2013).

21. Corti, D. et al. Heterosubtypic neutralizing antibodies are produced by individuals immunized with a seasonal influenza vaccine. J. Clin. Invest. 120, 1663 (2010).

22. Sangster, M. Y. et al. B cell response and hemagglutinin stalk-reactive antibody production in different age cohorts following 2009 H1N1 influenza virus vaccination. Clin. Vaccine Immunol. 20, 867-876 (2013).

23. Ellebedy, A. H. et al. Induction of broadly cross-reactive antibody responses to the influenza $\mathrm{HA}$ stem region following $\mathrm{H} 5 \mathrm{~N} 1$ vaccination in humans. Proc. Natl Acad. Sci. USA 111, 13133-13138 (2014).

24. Li, G. M. et al. Pandemic H1N1 influenza vaccine induces a recall response in humans that favors broadly cross-reactive memory B cells. Proc. Natl Acad. Sci. USA 109, 9047-9052 (2012)

25. Khurana, S., Frasca, D., Blomberg, B. \& Golding, H. AID activity in B cells strongly correlates with polyclonal antibody affinity maturation in-vivo following pandemic 2009-H1N1 vaccination in humans. Plos Pathog. 8, e1002920 (2012).

26. Wrammert, J. et al. Rapid cloning of high-affinity human monoclonal antibodies against influenza virus. Nature 453, 667 (2008).

27. Li, G. M. et al. Pandemic H1N1 influenza vaccine induces a recall response in humans that favors broadly cross-reactive memory B cells. Proc. Natl Acad. Sci. USA 109, 9047-9052 (2012).

28. DiLillo, D. J., Tan, G. S., Palese, P. \& Ravetch, J. V. Broadly neutralizing hemagglutinin stalk-specific antibodies require Fcgamma $\mathrm{R}$ interactions for protection against influenza virus in vivo. Nat. Med. 20, 143-151 (2014).
29. Corti, D. et al. A neutralizing antibody selected from plasma cells that binds to group 1 and group 2 influenza A hemagglutinins. Science 333, 850-856 (2011).

30. Tan, G. S. et al. A pan-H1 anti-hemagglutinin monoclonal antibody with potent broad-spectrum efficacy in vivo. J. Virol. 86, 6179-6188 (2012).

31. Jegaskanda, S. et al. Cross-reactive influenza-specific antibody-dependent cellular cytotoxicity in intravenous immunoglobulin as a potential therapeutic against emerging influenza viruses. J. Infect. Dis. 210, 1811-1822 (2014).

32. Cox, R. J. et al. An early humoral immune response in peripheral blood following parenteral inactivated influenza vaccination. Vaccine 12, 993 (1994).

33. Krammer, F., Pica, N., Hai, R., Margine, I. \& Palese, P. Chimeric hemagglutinin influenza virus vaccine constructs elicit broadly protective stalk-specific antibodies. J. Virol. 87, 6542-6550 (2013).

34. Pedersen, G. K. et al. Serum IgG titres, but not avidity, correlates with neutralizing antibody response after H5N1 vaccination. Vaccine 32, 4550-4557 (2014).

35. Jegaskanda, S. et al. Cross-reactive influenza-specific antibody-dependent cellular cytotoxicity antibodies in the absence of neutralizing antibodies. J. Immunol. 190, 1837 (2013).

\begin{abstract}
(c) (i)
This work is licensed under a Creative Commons Attribution 4.0 International License. The images or other third party material in this article are included in the article's Creative Commons license, unless indicated otherwise in the credit line; if the material is not included under the Creative Commons license, users will need to obtain permission from the license holder to reproduce the material. To view a copy of this license, visit http://creativecommons.org/licenses/ by/4.0/
\end{abstract}

(c) The Author(s) 2016

Supplementary Information accompanies the paper on the npj Vaccines website (http://www.nature.com/npjvaccines) 